\title{
Atezolizumab Caused Pityriasis Lichenoides-Like Drug Eruption Treated with Narrowband Ultraviolet B
}

Megan M Perez, BS ${ }^{1}$, Sebastian Otto-Meyer, BA ${ }^{1}$, Cuong V. Nguyen, MD¹, Lida Zheng, MD¹, Jennifer Choi, MD ${ }^{1}$, Lauren Guggina, MD ${ }^{2,3}$

1Department of Dermatology, Northwestern University Feinberg School of Medicine, Chicago, IL

${ }^{2}$ Harvard Medical School, Boston, MA

${ }^{3}$ Department of Dermatology, Brigham and Women's Hospital, Boston, MA

\section{ABSTRACT}

Pityriasis lichenoides is a rare cutaneous disease that exists along a spectrum with acute and chronic features. The acute form, pityriasis lichenoides et varioliformis acuta (PLEVA), presents as a sudden onset scaly and often crusted, erythematous papular eruption. The chronic form, pityriasis lichenoides chronica, presents similarly but with a more indolent onset. This inflammatory condition can have numerous triggers, including infections and medications. However, checkpoint inhibitors, despite being associated with a wide variety of cutaneous adverse events, have only rarely been associated with a pityriasis lichenoides-like eruption. We report a case of drug-induced pityriasis lichenoides-like eruption secondary to checkpoint inhibitor atezolizumab that was successfully treated with narrowband ultraviolet B (NBUVB) light. To our knowledge, this is the first case of an atezolizumabinduced pityriasis lichenoides which responded well to NBUVB.

\section{INTRODUCTION}

Pityriasis lichenoides is a rare cutaneous disease, which exists along a spectrum with acute and chronic features. ${ }^{1}$ The acute form, pityriasis lichenoides et varioliformis acuta (PLEVA), presents as an abrupt onset of pruritic, erythematous, scaly, often crusted, macules, papules and vesicles, on the trunk and extremities. Pityriasis lichenoides chronica (PLC) typically presents with a more indolent, scaling erythematous papular eruption. We report a case of atezolizumab induced pityriasis lichenoides, with both acute and chronic features, which cleared with narrowband ultraviolet $B$ (NBUVB) phototherapy treatment.

\section{CASE PRESENTATION}

A 68-year-old female with extensive-stage small cell lung cancer (SCLC) presented with two weeks of a pruritic skin eruption. She had been diagnosed with SCLC six months prior. She received four cycles of carboplatin/etoposide with the final dose approximately two months prior to presentation and palliative radiotherapy, followed by single agent atezolizumab every three weeks for maintenance therapy. After two doses of atezolizumab, she noted a diffuse pruritic eruption. Physical exam showed numerous red, scaling 2-10millimeter papules of the arms and trunk (Figure 1). 


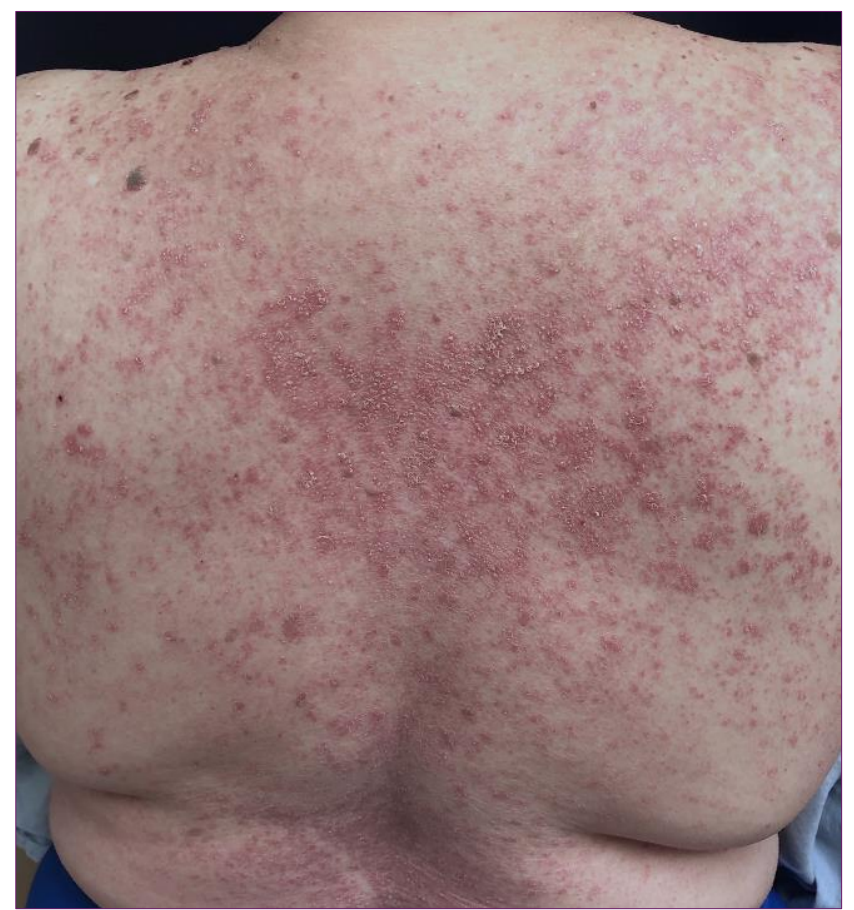

Figure 1. Pityriasis lichenoides: clinical photograph of back drug-induced variant of pityriasis lichenoides, presenting with numerous erythematous slightly scaly papules coalescing into a large plaque in the central upper back with a second large plaque visible on the lower back.

A punch biopsy of the arm showed a brisk interface process with overlying mounded parakeratosis alternating with an acute basket-weave stratum corneum. Vacuolar alteration with associated lymphocytic exocytosis and necrotic keratinocytes were noted along with rare eosinophils and red blood cell extravasation. These histopathologic features favored an acute presentation of pityriasis lichenoides (Figure 2). A drug-induced variant of pityriasis lichenoides was diagnosed secondary to atezolizumab. Initially, she was treated with topical clobetasol $0.05 \%$ ointment and oral prednisone initially dosed at $0.5 \mathrm{mg} / \mathrm{kg}$ with improvement of her skin lesions. However, her systemic steroid course was held when she developed hypoxemic respiratory failure due to a parainfluenza pneumonia with concerns for a concurrent aspergillus pneumonia, for which she was started on empiric voriconazole. Her eruption immediately flared off prednisone, though she continued topical clobetasol. A trial of minocycline was not tolerated due to nausea. She was ultimately initiated on NBUVB three times per week with clearance of her skin eruption after two months, followed by tapering off NBUVB therapy by 14 weeks. Although she initially experienced burning pain with NBUVB therapy, this resolved with discontinuation of her voriconazole. Her skin remains clear one year after her initial presentation. Her SCLC is stable with partial response 14 months after holding immunotherapy and she continues active surveillance with oncology.

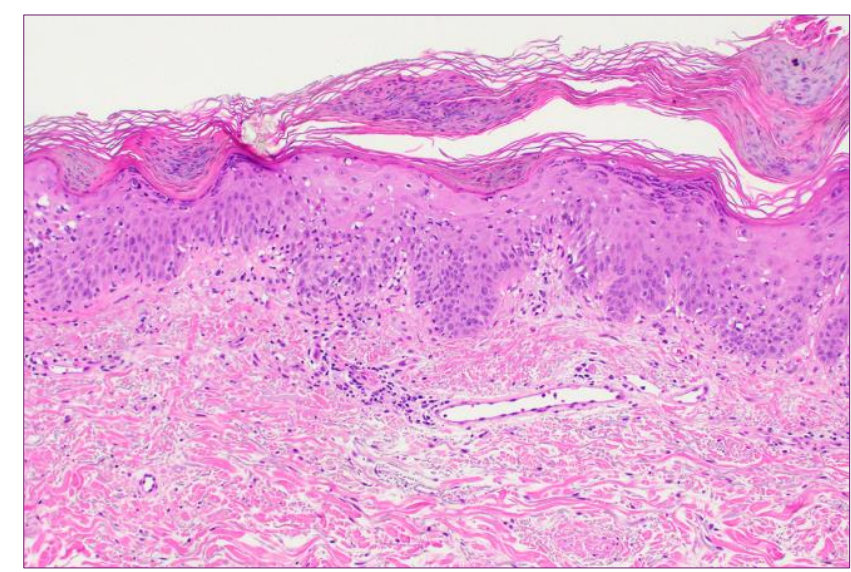

Figure 2. Histopathological features of pityriasis lichenoides from punch biopsy of arm shows a basketweave stratum corneum with serous crust and mounded parakeratosis. There is a robust vacuolar interface process with many necrotic keratinocytes and exocytosis of lymphocytes and red blood cells. The infiltrate is predominantly lymphohistiocytic with a rare eosinophil noted, consistent with a diagnosis of drug-induced pityriasis lichenoides, favoring the acute presentation, PLEVA. (H\&E, 100x)

\section{DISCUSSION}

Atezolizumab is a programmed death-ligand1 (PD-L1) inhibitor approved for treatment of multiple cancers. ${ }^{2}$ Immune checkpoint inhibitors have been reported to cause numerous cutaneous eruptions including 
lichenoid reactions, psoriasiform eruptions, vitiligo, eczema, subacute cutaneous lupus erythematosus (SCLE), and bullous pemphigoid. ${ }^{3}$ There is one report of PLC-like drug eruption with another immune checkpoint inhibitor, pembrolizumab, during treatment of a 67-year-old woman with melanoma, which cleared with topical steroids. ${ }^{4}$ Notably, our patient presented with more extensive distribution and increased confluence of the lesions along with severe associated pruritus and pathological findings more suggestive of PLEVA. Our patient had a minimal response to topical steroids, and oral steroids were not a viable treatment option due to concerns for a fungal pneumonia.

When considering treatments for drug induced pityriasis lichenoides, our patient presented unique therapeutic challenges given her complicated medical history. Given her known extensive stage SCLC and possible fungal pneumonia, treatment options were limited. First line treatments include topical treatments for pityriasis lichenoides include topical steroids and, less frequently, topical calcineurin inhibitors. ${ }^{1}$ However, treatment clearance (full or partial) for topical steroids is fifty percent or less in most case series and evidence for topical calcineurin inhibitors is based off of case reports. ${ }^{1,5}$ Minocycline was initially chosen as a steroid sparing agent given our patients comorbidities. Although a wide range of systemic agents have been used, most commonly antibiotics such as minocycline, tetracycline, and erythromycin are employed, with their success likely due to antiinflammatory properties. Limited case series and reports indicate slightly higher efficacy than topical treatments alone, with most reporting a complete or partial response in the large majority of patients. ${ }^{1}$ Systemic immunosuppressants such as corticosteroids or methotrexate are reserved for severe or recalcitrant cases, which were ultimately contraindicated given our patients ongoing infectious complications. NBUVB is an additional common treatment that may be used provided a patient does not have a medical contraindication, which include a history of light sensitive conditions such as lupus erythematosus or xeroderma pigmentosa. ${ }^{6}$ Fortunately, our patient responded well to this therapy.

To our knowledge this is the first reported case of an immune checkpoint inhibitor induced pityriasis lichenoides which responded well to NBUVB. It is also the first case with reported acute pityriasis lichenoides features. NBUVB has previously shown efficacy in PLEVA and PLC. ${ }^{1}$ A retrospective study of PLEVA patients $(n=23)$ found complete response to NBUVB in $65.2 \%$ of patients after $30-53$ sessions, with all patients experiencing some response. ${ }^{7}$ Reassuringly, only two patients experienced relapse with a mean follow-up time of 6.9 months. However, these findings cannot be fully extrapolated to our case, as they concern individuals with PLEVA often lasting years without a clear drug trigger noted.

A final important consideration with NBUVB therapy this case raised, is the significant pain with phototherapy our patient experienced during concomitant voriconazole treatment, It is important to recognize that photosensitizing medications, such as voriconazole, may cause significant photosensitivity and likely resulted in the burning sensation during NBUVB. ${ }^{8}$ Indeed, voriconazole is one of the most commonlyreported drugs associated with photosensitivity. Appropriate counseling and discontinuation of therapy may be necessary in patients who experience these effects. We share this unique case to encourage the consideration of NBUVB therapy for pityriasis 
lichenoides-like drug eruptions due to checkpoint inhibitors.

\author{
Abbreviations \\ PLEVA - Pityriasis lichenoides et varioliformis acuta \\ NBUVB - Narrowband ultraviolet B \\ SCLC - Small cell lung cancer \\ PD-L1 - Program death ligand-1 \\ SCLE - Subacute cutaneous lupus erythematosus
}

Conflict of Interest Disclosures: None

Funding: None

\section{Corresponding Author:}

Lauren Guggina, MD

Department of Dermatology

Brigham and Women's Hospital

221 Longwood Avenue

Boston, MA 02115

Email: Iguggina@bwh.harvard.edu
8. 8. Kim WB, Shelley AJ, Novice K, Joo J, Lim HW, Glassman SJ. Drug-induced phototoxicity: A systematic review. Journal of the American Academy of Dermatology. 2018;79(6):1069-1075.

\section{References:}

1. Bellinato F, Maurelli M, Gisondi P, Girolomoni G. A systematic review of treatments for pityriasis lichenoides. Journal of the European Academy of Dermatology and Venereology : JEADV. 2019;33(11):2039-2049.

2. 2. Akinleye $A$, Rasool Z. Immune checkpoint inhibitors of PD-L1 as cancer therapeutics. Journal of hematology \& oncology. 2019;12(1):92.

3. 3. Sibaud V. Dermatologic Reactions to Immune Checkpoint Inhibitors : Skin Toxicities and Immunotherapy. American journal of clinical dermatology. 2018;19(3):345-361.

4. 4. Mutgi KA, Milhem M, Swick BL, Liu V. Pityriasis lichenoides chronica-like drug eruption developing during pembrolizumab treatment for metastatic melanoma. JAAD case reports. 2016;2(4):343-345.

5. 5. Wahie S, Hiscutt E, Natarajan S, Taylor A. Pityriasis lichenoides: the differences between children and adults. Br J Dermatol. 2007; 157(5):941-945.

6. 6. Ultraviolet Phototherapy Management of Moderate-to-Severe Plaque Psoriasis: An Evidence-Based Analysis. Ont Health Technol Assess Ser. 2009;9(27):1-66.

7. 7. Aydogan $\mathrm{K}$, Saricaoglu $\mathrm{H}$, Turan $\mathrm{H}$. Narrowband UVB (311 nm, TL01) phototherapy for pityriasis lichenoides. Photodermatology, photoimmunology \& photomedicine. 2008;24(3):128-133. 